\title{
PDNA-encoding Emm55 Autologous Cancer Cell Vaccine IFx-Hu2.0
}

National Cancer Institute

\section{Source}

National Cancer Institute. pDNA-encoding Emm55 Autologous Cancer Cell Vaccine IFX-

Hu2.0. NCl Thesaurus. Code C157262.

A whole cell cancer vaccine composed of irradiated autologous whole tumor cells that are transfected, ex vivo, with a plasmid DNA encoding the highly immunogenic Streptococcus pyogenes (S. pyogenes) bacterial antigen Emm55, with potential immunostimulating and antineoplastic activities. Upon intralesional administration of IFXHu2.0, the tumor cells expressing the Emm55 bacterial antigen on their cell surface are taken up and processed by antigen-presenting cells (APCs), thereby presenting both the Emm55 and the patient-specific tumor-associated antigens (TAAs) to the immune system. This activates the immune system to elicit a tumor antigen-specific cytotoxic Tlymphocyte (CTL)-mediated immune response against the tumor cells expressing the TAAs. 